\title{
DNA Hybridization Detection Based on Resonance Frequency Readout in Graphene on Au SPR Biosensor
}

\author{
Md. Biplob Hossain ${ }^{1}$ and Md. Masud Rana ${ }^{1,2}$ \\ ${ }^{1}$ Department of Electrical and Electronic Engineering (EEE), Rajshahi University of Engineering and Technology (RUET), \\ Rajshahi 6204, Bangladesh \\ ${ }^{2}$ Faculty of Engineering and IT, University of Technology Sydney (UTS), Sydney, NSW 2007, Australia
}

Correspondence should be addressed to Md. Biplob Hossain; biplobh.eee10@gmail.com

Received 27 August 2015; Revised 19 October 2015; Accepted 25 October 2015

Academic Editor: Zhi-Mei Qi

Copyright (c) 2016 Md. B. Hossain and Md. M. Rana. This is an open access article distributed under the Creative Commons Attribution License, which permits unrestricted use, distribution, and reproduction in any medium, provided the original work is properly cited.

\begin{abstract}
This paper demonstrates a numerical modeling of surface plasmon resonance (SPR) biosensor for detecting DNA hybridization by recording the resonance frequency characteristics (RFC). The proposed sensor is designed based on graphene material as biomolecular recognition elements (BRE) and the sharp SPR curve of gold (Au). Numerical analysis shows that the variation of RFC for mismatched DNA strands is quiet negligible whereas that for complementary DNA strands is considerably countable. Here, graphene is used to perform faster immobilization between target DNA and probe DNA. The usage of graphene also changes the RFC that ensure hybridization of DNA event by utilizing its optochemical property. In addition, proposed sensor successfully distinguishes between hybridization and single-nucleotide polymorphisms (SNP) by observing the variation level of RFC and maximum transmittance. Therefore, the proposed frequency readout based SPR sensor could potentially open a new window of detection for biomolecular interactions. We also highlight the advantage of using graphene sublayer by performing the sensitivity analysis. Sandwiching of each graphene sublayer enhances $95 \%$ sensitivity comparing with conventional SPR sensor.
\end{abstract}

\section{Introduction}

DNA hybridization is a biochemical process to find out the DNA nucleotide bonding in proper orientation (between adenine $(\mathrm{A})$ and thymine $(\mathrm{T})$ or between guanine $(\mathrm{G})$ and cytosine (C)) or in mutated sequence shown in Figure 4, in [1]. DNA mutation is a piece of information that indicates hepatitis B [2], cancer, and other complex congenital diseases [1]. Nowadays, biosensors have been intensely researched owing to their importance for many industry applications such as medical diagnostics, enzyme detection, food safety, and environmental monitoring $[3,4]$. Today, a number of biosensor schemes have been developed; among them fiberoptic surface plasmon resonance (SPR) biosensor bears the advantages of compactness, light weight, high sensitivity, the ease of multiplexing and remote sensing, and so forth [5]. Fiber-optic surface plasmon resonance (SPR) biosensor is an evanescent guided electromagnetic wave mode [6] that propagates along a metal-dielectric interface (e.g., gold and biomolecule) by utilizing the surface plasmon waves (SPW) [5]. The variation of the biomolecules concentration will produce the local change of the surrounding refractive index (SRI) near the sensor surface that results in changing the propagation constant of the SPW and thus the resonance frequency [5]. The SPR technique has been successfully applied in various fields, such as chemical and biochemical sensing, film characterization, and beam polarization selection [7]. In biochemical sensing applications, SPR is used for DNA-DNA hybridization [1,5-8], protein-protein $[9,10]$, protein-DNA $[11,12]$, enzyme-substrate or inhibitor $[11,13]$, receptor-drug $[14,15]$, lipid membrane-protein $[16$, 17], protein-polysaccharide [18], cell or virus-protein [19-21], and so forth.

In this paper, a numerical modeling of graphene based SPR biosensor is proposed for DNA hybridization detection which results in faster immobilization by monitoring the resonance frequency characteristics (RFC). Since graphene has outstanding properties such as high surface area [22], 


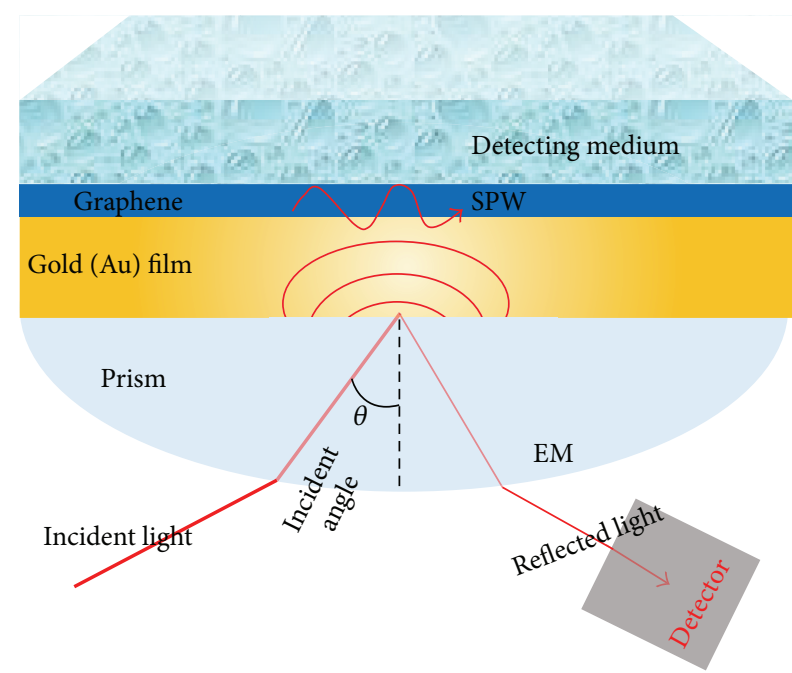

(a)

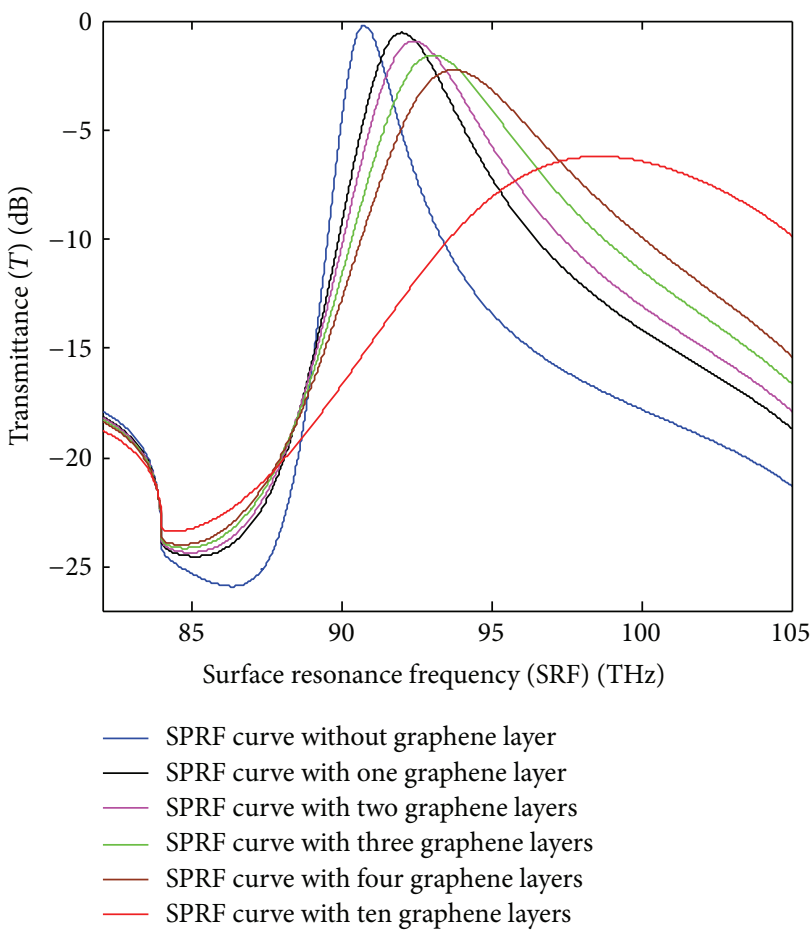

(b)

Figure 1: (a) Schematic of the 4-layerd model for surface plasmon resonance (SPR) biosensor: prism, Au (50 nm), graphene (0.34L nm, where $L$ is the number of graphene sublayers), and sensing medium (water). (b) The $T \sim$ SRF curves for the conventional biosensor $(L=0)$ and graphene coated biosensor (for $L=1,2, \ldots, 10$ ) before the adsorption of DNA molecules.

electrical conductivity [23], and biocompatibility [1, 23], so graphene monolayer is sandwiched between metal films and sensing medium as biomolecular recognition elements (BRE) for enhancing sensor sensitivity $95 \%$ better than the conventional sensitivity. Carrier concentration is varied due to the immobilization of DNA molecule on the sensor surface that changes the refractive index (RI) near the graphene and gold $(\mathrm{Au})$. The RI change will in turn lead to change in the SPR angle that translates a change in propagation constant of SPW. Moreover, graphene layer on gold will also modify the propagation constant of SPW, thereby varying the sensitivity due to RI change.

\section{Numerical Modeling and Sensor Methodology}

We introduce a numerical model using Kretschmann prism arrangement $[8,24]$ described in Figure 1(a) that shows the schematic configuration of the proposed graphene-on-gold surface plasmon resonance biosensor, where the first layer is a SF10 glass prism (RI $\left.n_{p}=1.723\right)$, second layer is gold film (thickness and RI are $d_{\mathrm{Au}}=50 \mathrm{~nm}$ and $n_{\mathrm{Au}}=0.1726+i 3.4218$, resp. [8]), third layer is graphene (thickness and RI are $d_{G}=$ $0.34 \mathrm{~nm}$ and $n_{G}=3+i 1.149106$, resp. [8]), and the final layer is water (RI $\left.n_{s}=1.33[24]\right)$. Then, a TM polarized He-Ne (wavelength, $\lambda=633 \mathrm{~nm}$ ) light wave is used, which passes through the prism and some portion is reflected at the prismgold interface.
While impinging light energy to prism-gold interface, an evanescent wave is generated which is known as surface plasmon wave (SPW) that propagates with the different propagation constant from optical wave which is defined in $[8,25]$. The propagation constant of SPW can be adjusted to be equal to the propagation constant of optical wave that is also defined in [8]. The point at which optical wave propagation constant equals SPW propagation constant is called surface plasmon resonance point (SPRP) [8]. Equation (1) suggests that SPR angle is a dependent parameter on RI of sensing medium. At SPRP, the frequency at which SPW propagates is called surface resonance frequency (SRF) and the angle of incidence is called SPR angle that can be given in [25] shown as follows:

$$
\theta_{\mathrm{SPR}}=\sin ^{-1} \sqrt{\frac{n_{\mathrm{Au}}^{2} n_{s}^{2}}{n_{p}^{2}\left(n_{\mathrm{Au}}^{2}+n_{s}^{2}\right)}} .
$$

When submersing single strand target DNA (t-DNA) into probe DNA (p-DNA) on the sensor surface, then the RI of sensing medium is mathematically modified as $[26,27]$

$$
n_{s}^{d}=n_{s}+c_{a} \frac{d n}{d c} .
$$

Here, $n_{s}^{d}$ is the RI of the sensing dielectric after adsorption of DNA molecules, $n_{s}$ is RI of the sensing dielectric before adsorption of DNA molecules, $c_{a}$ is the concentration of 
adsorbed DNA molecules, and $d n / d c$ is the RI increment due to the adsorbate (DNA). The RI increment parameter is $d n / d c=0.153 \mathrm{~cm}^{3} / \mathrm{gm}$ in the case of water [26, 27]. If SPR angle changes, the propagation constant of SPW also changes which has been explained in the literature $[1,8,28,29]$ as given in the following:

$$
k_{\mathrm{SPW}}=\frac{2 \pi}{\lambda} n_{p} \sin \theta_{\mathrm{SPR}}
$$

and finally if propagation constant of SPW changes it makes the surface resonance frequency (SRF) change which can be explained by the following equation:

$$
\mathrm{SRF}=\frac{c_{0}}{n_{\mathrm{geo}}} \frac{k_{\mathrm{SPW}}}{2 \pi},
$$

where $c_{0} / n_{\text {geo }}$ is the propagation velocity of SPW that is a perpendicularly confined evanescent electromagnetic wave $[6,8]$ and $n_{\text {geo }}$ is the geometric mean of interface between gold and sensing medium $\left(n_{\text {geo }}=\sqrt[3]{n_{\mathrm{Au}} n_{s} n_{G}}\right.$, omitting imaginary part that is essential to real world design [25]) linked by graphene because SPW propagates at the interface between the metal and the dielectric (i.e., sensing medium) $[6,8]$. If the incident angle of optical wave is tuned, SPR condition is achieved in which reflectance $(R)$ of reflected wave is minimum and transmittance $(T)$ is maximum and then SPW penetrate at SPF along the $x$-direction. We define a plot "transmittance versus surface resonance frequency (T SRF)," as surface resonance frequency response (SRFR) curve. To generate a SPFR curve, we used Fresnel equations [30] for four-layered heterooptical system to determine transmitted light intensity [25].

\section{Numerical Results}

At first numerical analysis is presented by comparing the $T \sim$ SRF characteristics curve in between with graphene sublayer and without graphene sublayer before adding DNA molecule which is normally known as bare sensor, as depicted in Figure 1(b). The SPR frequency without graphene is $91.70 \mathrm{THz}$ and with single layer of graphene is $92.65 \mathrm{THz}$. The SPR frequency is increased $(\triangle \mathrm{SRF})$ to $0.95 \mathrm{THz}$ in the right side of the $T \sim S R F$ curve due to sandwiching each graphene film whose phenomenon accounts for enhanced sensitivity [5, 8] described in the sensitivity analysis section. The change of detecting attributors $\left(\triangle \mathrm{SRF}\right.$ and $T_{\max }$ ) due to adding graphene film is provided in Table 1. The data of Table 1 has been extracted from Figure 1(b) where results without graphene layer are used as reference in order to calculate other $\triangle \mathrm{SRF}$ and $T_{\max }$.

In our SPR device, water is used as sensing medium that helps to measure the dependency of transmittance on SRF. Further work is continued by assuming that our sensor is susceptible of differentiating between complementary and single-based mismatched DNA with regard to the analysis of mutations and polymorphisms [1]. Since the change of concentration is due to the immobilization of DNA within the sensing medium, the local RI $\left(n_{s}\right)$ of the sensing medium is also changed described by (2). From (1) we know that
TABLE 1: $T_{\max }$ and SRF with graphene film and without graphene film.

\begin{tabular}{lccc}
\hline Graphene layer & $T_{\max }[\mathrm{dB}]$ & SRF $[\mathrm{THz}]$ & $\Delta$ SRF $[\mathrm{THz}]$ \\
\hline No layer $(L=0 \mathrm{~nm})$ & 0.2267 & 91.70 & 0.00 \\
One layer $(L=1 * 0.34 \mathrm{~nm})$ & 0.5494 & 92.65 & 0.95 \\
Two layers $(L=2 * 0.34 \mathrm{~nm})$ & 0.9400 & 93.80 & 1.90 \\
Three layers $(L=3 * 0.34 \mathrm{~nm})$ & 1.5870 & 94.95 & 2.85 \\
Four layers $(L=4 * 0.34 \mathrm{~nm})$ & 2.2630 & 95.10 & 3.80 \\
Ten layers $(L=10 * 0.34 \mathrm{~nm})$ & 6.2180 & 101.20 & 9.50 \\
\hline
\end{tabular}

SPR angle changes if $n_{s}$ change which finally translates $k_{\mathrm{SPW}}$ change observed from (3). Then, at the transition point, where the SPW wavevector and optic wavevector are equal to each other, the maximum transmittance $\left(T_{\max }\right)$ is detected.

This point is called SPRP which is mentioned in Section 2. It is observed from Figure 2(b), by immobilization of the probe DNA (p-DNA), either complementary or mismatched, on the graphene surface, the SPRP is considerably right shifted by $1.15 \mathrm{THz}$. This phenomenon can demonstrate the dependency of SRF on the immobilization of the probe DNA and hybridization of the complementary target DNA (C-tDNA) [1]. Introducing DNA as electron-rich molecules, the number of carriers would change the graphene concentration which has let to varying the propagation constant. Finally, the hybridization event has been performed by adding complementary DNA sequences $[1,31]$.

As illustrated in Figure 3, single-based mismatched combination occurred with the submersion of the noncomplementary t-DNA to the immobilized capture probe on SPR device; then no significant change of T SRF characteristics resulted (change of SRF angle reference presenting mismatched target is $\triangle \mathrm{SRF}=0.1 \mathrm{THz}$ ) due to its nonbonding reaction between two pairs of DNA strands since they cannot be hybrid. In this case we conclude that there are no associated charges with the target molecule that can incorporate an obvious change to the applied sensing dielectrics. It is also observed that the SPR device specifically recognizes the target DNA sequences. In light of this fact, the focus of this paper is to present a new strategy for DNA sensor with the capability of detection of SNP.

Figure 4(b) describes the T SRF characteristics for different concentrated complementary target DNA (C-t-DNA), where each color line depicts finite concentration of the DNA molecules. According to the numerical data, SRF and $T_{\max }$ have acted as hybridization detectors. These parameters will vary and decision will be made based on these variations when complementary DNA molecules are combined with probe.

SRF and $T_{\max }$ are calculated for different concentrated C-t-DNA and tabulated in Table 2, in order to know how these parameters would change with changing the concentrations. A considerable increase of SRF that determines DNA hybridization is pointedly seen. The numerical data apprises the strong dependency of the SRF on the concentration increment that reflects in the T SRF characteristics curve. With DNA hybridization, the C-t-DNA molecules 


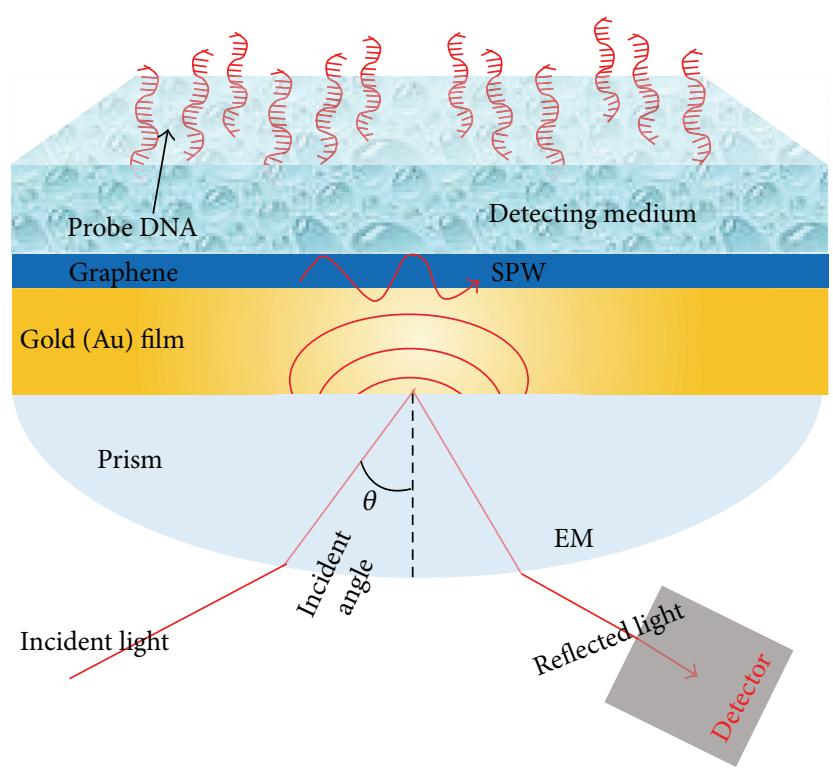

(a)

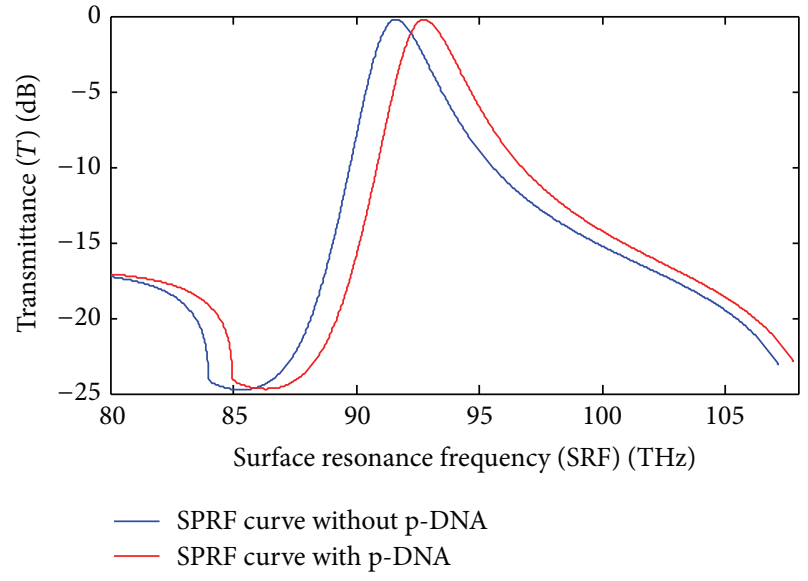

(b)

FiguRE 2: (a) Schematic of initial stage detection concept. (b) Surface resonance frequency response curves before and after the adsorption of biomolecules, blue line for bare sensor and red line for sensor with $1000 \mathrm{nM}$ probe DNA.

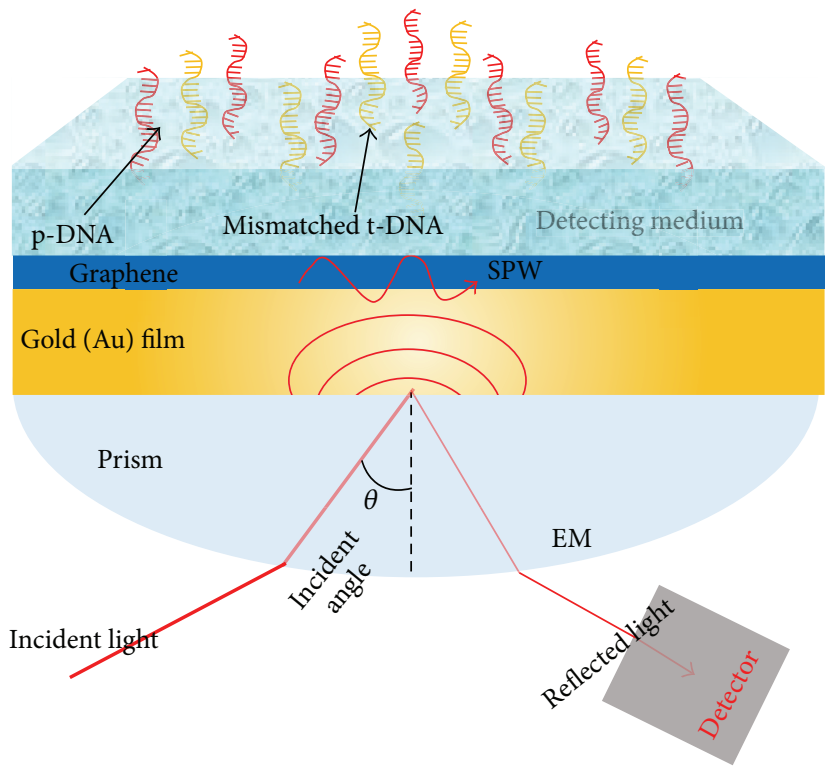

(a)

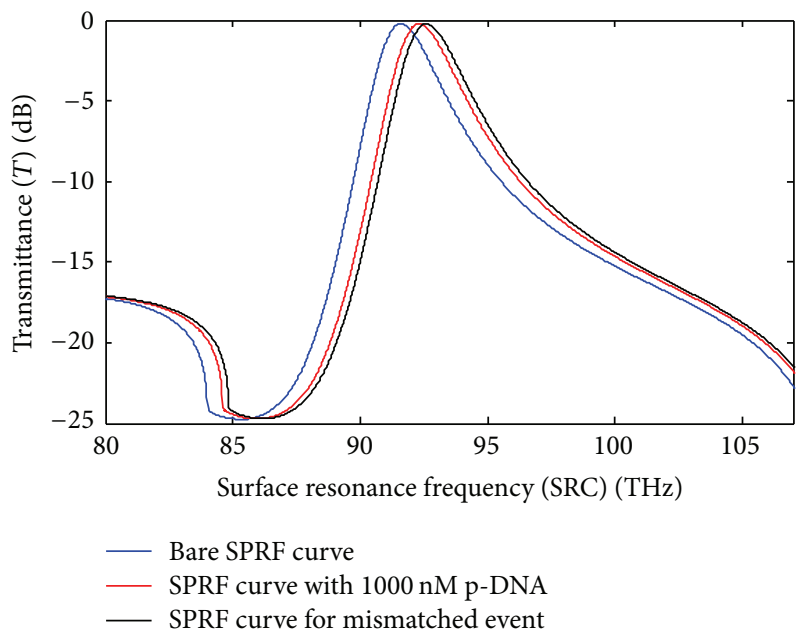

(b)

FIGURE 3: (a) Schematic configuration of mismatched event in SPR sensor. (b) Comparison of SPRF curve before adding DNA molecules (bare sensor, blue line) and after adding p-DNA (red line) and after adding mismatched t-DNA (black line).

are reacted with the graphene surface, commonly known as "graphene-nucleotide interaction" and generated n-doping effect which is liable to turn right of SPRP. It is stated that the interaction between nucleotide and graphene surface upon DNA hybridization has an enormous influence on SRF too, which can change it rightwards [32]. For clarifying DNA hybridization, $\Delta \mathrm{SRF}_{p-t}$ and $\Delta T_{\max }^{p-t}$ are represented as the detecting attributors. The following equations are used to determine these attributors:

$$
\begin{aligned}
\left(\Delta \mathrm{SRF}_{p-t}\right)_{\min } & =\left|\mathrm{SRF}_{p}-\mathrm{SRF}_{t=1000 \mathrm{nM}}\right|=0.72 \mathrm{THz}, \\
\left(\Delta T_{\max }^{p-t}\right)_{\min } & =\left|T_{\max }^{p}-T_{\max }^{t=1000 \mathrm{nM}}\right|=0.003 \mathrm{~dB} .
\end{aligned}
$$




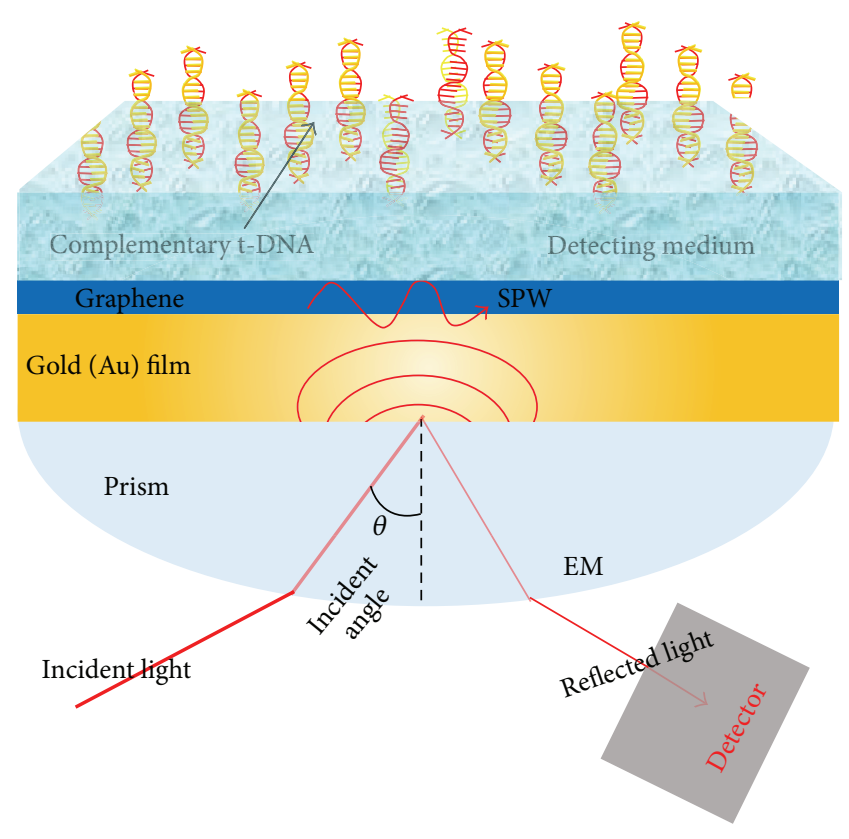

(a)

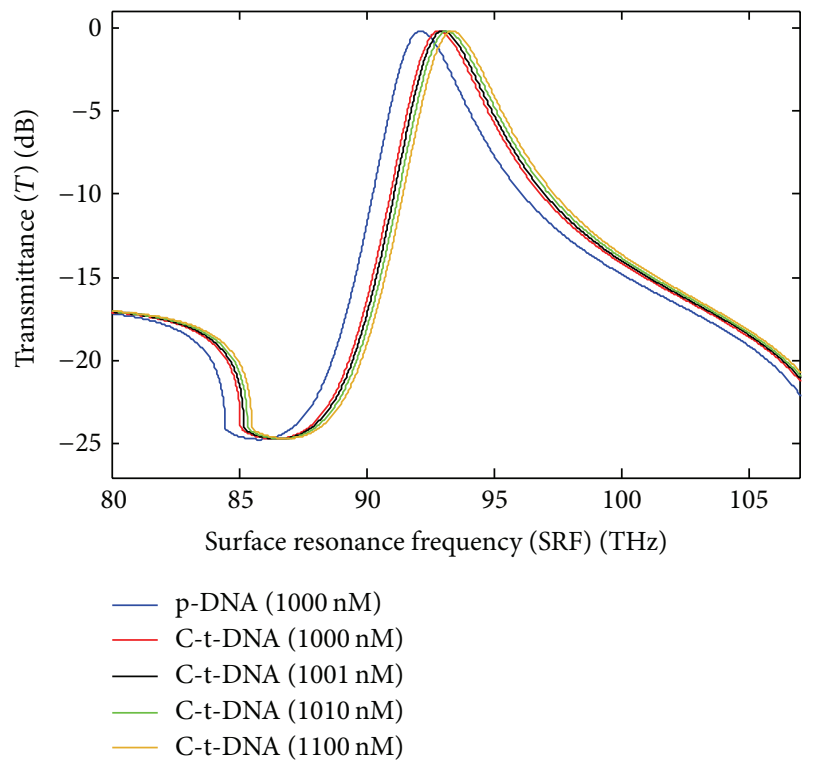

(b)

FIGURE 4: Immersing complementary DNA solution. (a) Schematic after immersing the device in complementary DNA solution and (b) $T \sim$ SRF characteristics response for different concentrated C-t-DNA.

TABLE 2: $\Delta T_{\max }^{p-t}$ and $\Delta \mathrm{SRF}_{p-t}$ for different concentration of DNA molecules.

\begin{tabular}{lcc}
\hline $\begin{array}{l}\text { Concentration } \\
\left(c_{a}\right)[\mathrm{nM}]\end{array}$ & $\begin{array}{c}\Delta T_{\max }^{p-t}[\mathrm{~dB}]= \\
\left|T_{\max }^{p}-T_{\max }^{t}\right|\end{array}$ & $\begin{array}{c}\Delta \mathrm{SRF}_{p-t}[\mathrm{THz}]= \\
\left|\mathrm{SRF}_{p}-\mathrm{SRF}_{t}\right|\end{array}$ \\
\hline 1000 (target) & 0.0030 & 0.72 \\
1001 (target) & 0.0043 & 0.87 \\
1010 (target) & 0.0052 & 1.05 \\
1100 (target) & 0.0065 & 1.25 \\
\hline
\end{tabular}

In (5a) and (5b) $\mathrm{SRF}_{p}, \mathrm{SRF}_{t}, T_{\text {max }}^{p}$, and $T_{\max }^{t}$ represent the SRF of $\mathrm{p}$-DNA, SRF of specific concentrated C-t-DNA, maximum transmittance of $\mathrm{p}$-DNA, and specific concentrated C-tDNA, respectively. Finally taking advantage of the attributor's values, a decision making table (Table 3 ) is prepared and can be utilized. When the change of $\Delta S R F$ and $\Delta T_{\max }^{p-t}$ is greater than or equal to $(\Delta \mathrm{SRF})_{\min }(0.72 \mathrm{THz})$ and $\left(\Delta T_{\max }^{p-t}\right)_{\min }$ $(0.003 \mathrm{~dB})$, then DNA-DNA hybridization is ensued and if the change of $\Delta \mathrm{SRF}$ and $\Delta T_{\max }^{p-t}$ is less than $(\Delta \mathrm{SRF})_{\min }$ and $\left(\Delta T_{\max }^{p-t}\right)_{\min }$, then SNP happens; except both of these cases, no effective result will be established, and detection procedure should be restarted.

\section{Sensitivity Analysis}

The sensitivity of the proposed optical sensor is defined as the ratio of the SRF change, $\Delta \mathrm{SRF}^{T}$ (sensor output), to the change
TABLE 3: Decision making table based upon different conditions which happened to detective parameters.

\begin{tabular}{lllc}
\hline Conditions & & Decision \\
\hline$\Delta T_{\max }^{p-t} \geq\left(\Delta T_{\max }^{p-t}\right)_{\min }$ & $\& \&$ & $\Delta \mathrm{SRF}_{p-t} \geq\left(\Delta \mathrm{SRF}_{p-t}\right)_{\min }$ & $\begin{array}{c}\text { Hybridization } \\
\text { happened }\end{array}$ \\
$\Delta T_{\max }^{p-t} \geq\left(\Delta T_{\max }^{p-t}\right)_{\min }$ & $\& \&$ & $\Delta \mathrm{SRF}_{p-t} \leq\left(\Delta \mathrm{SRF}_{p-t}\right)_{\min }$ & Try again \\
$\Delta T_{\max }^{p-t} \leq\left(\Delta T_{\max }^{p-t}\right)_{\min }$ & $\& \&$ & $\Delta \mathrm{SRF}_{p-t} \geq\left(\Delta \mathrm{SRF}_{p-t}\right)_{\min }$ & Try again \\
$\Delta T_{\max }^{p-t}<\left(\Delta T_{\max }^{p-t}\right)_{\min }$ & $\& \&$ & $\Delta \mathrm{SRF}_{p-t}<\left(\Delta \mathrm{SRF}_{p-t}\right)_{\min }$ & $\begin{array}{c}\text { SNP } \\
\text { happened }\end{array}$ \\
\hline
\end{tabular}

in concentration of biomolecules, $\Delta c_{a}$ (sensor input), as given in the following:

$$
\begin{aligned}
S^{G} & =\frac{\Delta \mathrm{SRF}^{T}}{\Delta c_{a}}=\frac{\Delta \mathrm{SRF}^{0}+\Delta \mathrm{SRF}^{L}}{\Delta c_{a}} \\
& =\frac{\Delta \mathrm{SRF}^{0}+L \Delta \mathrm{SRF}^{L=1}}{\Delta c_{a}}=\frac{\Delta \mathrm{SRF}^{0}+0.95 L \Delta \mathrm{SRF}^{0}}{\Delta c_{a}} \\
& =(1+0.95 L) \frac{\Delta \mathrm{SRF}^{0}}{\Delta c_{a}} \\
\text { or } S^{G} & =(1+0.95 L) S^{0}
\end{aligned}
$$

where $\triangle \mathrm{SRF}^{T}$ is the SRF change of sensor summing with and without graphene sublayers, $S^{0}$ is the sensitivity of the devices without graphene film, and $S^{G}$ is the sensitivity for different number of graphene sublayers $(L=1,2,3, \ldots)$. The study 
on graphene sandwiched SPR sensor ensures $1+0.95 L$ times higher sensitivity. If single graphene sublayer is considered then sensitivity has increased to $95 \%$ for DNA detection which is a great achievement compared to other results that had been $34 \%$ in [8]. The sensitivity has been improved due to the changes of detecting attributors. In this study, we consider surface resonance frequency and maximum transmittance as the detecting attributors, whereas, in [8], surface resonance angle and minimum reflectance are considered for detecting attributors.

\section{Conclusion}

In this paper, a numerical modeling of SPR biosensor is proposed for detecting DNA-DNA hybridization by monitoring the resonance frequency characteristics (RFC). The proposed biosensor has been designed based on graphene material which is sandwiched between metal film and sensing medium for enhancing sensor sensitivity. Without graphene sublayers SPR sensor provides slower immobilization between target DNA and probe DNA as well as lesser sensitivity which are the causes of poor efficiency. It is observed that $95 \%$ sensitivity has been increased for adding each sublayer if surface resonance frequency and maximum transmittance are chosen as the detecting attributors.

\section{Conflict of Interests}

The authors declare that there is no conflict of interests regarding the publication of this paper.

\section{References}

[1] H. Karimi, R. Yusof, R. Rahmani, H. Hosseinpour, and M. T. Ahmadi, "Development of solution-gated graphene transistor model for biosensors," Nanoscale Research Letters, vol. 9, no. 1, pp. 1-11, 2014.

[2] C.-W. Huang, Y.-J. Huang, T.-H. Lin et al., "An integrated microcantilever-based wireless DNA chip for Hepatitis B Virus (HBV) DNA detection," in Proceedings of the 15th International Conference on Miniaturized Systems for Chemistry and Life Sciences (MicroTAS '11), pp. 984-986, Seattle, Wash, USA, October 2011.

[3] M. Pumera, "Graphene in biosensing," Materials Today, vol. 14, no. 7-8, pp. 308-315, 2011.

[4] J. Homola, "Present and future of surface plasmon resonance biosensors," Analytical and Bioanalytical Chemistry, vol. 377, no. 3, pp. 528-539, 2003.

[5] H. Fu, S. Zhang, H. Chen, and J. Weng, "Graphene enhances the sensitivity of fiber-optic surface plasmon resonance biosensor," IEEE Sensors Journal, vol. 15, no. 10, pp. 5478-5482, 2015.

[6] N.-F. Chiu, W.-C. Lee, and T.-S. Jiang, "Constructing a novel asymmetric dielectric structure toward the realization of highperformance surface plasmon resonance biosensors," IEEE Sensors Journal, vol. 13, no. 9, pp. 3483-3489, 2013.

[7] T.-J. Wang, C.-W. Tu, and F.-K. Liu, "Integrated-optic surfaceplasmon-resonance biosensor using gold nanoparticles by bipolarization detection," IEEE Journal on Selected Topics in Quantum Electronics, vol. 11, no. 2, pp. 493-499, 2005.
[8] L. Wu, H. S. Chu, W. S. Koh, and E. P. Li, "Highly sensitive graphene biosensors based on surface plasmon resonance," Optics Express, vol. 18, no. 14, pp. 14395-14400, 2010.

[9] M. Kim, K. Park, E.-J. Jeong, Y.-B. Shin, and B. H. Chung, "Surface plasmon resonance imaging analysis of protein-protein interactions using on-chip-expressed capture protein," Analytical Biochemistry, vol. 351, no. 2, pp. 298-304, 2006.

[10] A. Madeira, E. Vikeved, A. Nilsson, B. Sjögren, P. E. Andrén, and P. Svenningsson, "Identification of protein-protein interactions by surface plasmon resonance followed by mass spectrometry," Current Protocols in Protein Science, vol. 65, pp. 19.21.1-19.21.9, 2011.

[11] J. Majka and C. Speck, "Analysis of protein-DNA interactions using surface plasmon resonance," Advances in Biochemical Engineering/Biotechnology, vol. 104, pp. 13-36, 2007.

[12] H. F. Teh, W. Y. X. Peh, X. Su, and J. S. Thomsen, "Characterization of protein-DNA interactions using surface plasmon resonance spectroscopy with various assay schemes," Biochemistry, vol. 46, no. 8, pp. 2127-2135, 2007.

[13] C.-C. Fong, W.-P. Lai, Y.-C. Leung, S. C.-L. Lo, M.-S. Wong, and M. Yang, "Study of substrate-enzyme interaction between immobilized pyridoxamine and recombinant porcine pyridoxal kinase using surface plasmon resonance biosensor," Biochimica et Biophysica Acta, vol. 1596, no. 1, pp. 95-107, 2002.

[14] Z. Salamon, S. Cowell, E. Varga, H. I. Yamamura, V. J. Hruby, and G. Tollin, "Plasmon resonance studies of agonist/antagonist binding to the human delta-opioid receptor: new structural insights into receptor-ligand interactions," Biophysical Journal, vol. 79, no. 5, pp. 2463-2474, 2000.

[15] R. L. Rich, L. R. Hoth, K. F. Geoghegan et al., "Kinetic analysis of estrogen receptor/ligand interactions," Proceedings of the National Academy of Sciences of the United States of America, vol. 99, no. 13, pp. 8562-8567, 2002.

[16] O. L. Baron, D. Pauron, and S. Antipolis, "Protein-lipid interaction analysis by surface plasmon resonance (SPR)," Bio-Protocol, vol. 4, no. 18, pp. 1-8, 2014.

[17] E.-M. Erb, X. Chen, S. Allen et al., "Characterization of the surfaces generated by liposome binding to the modified dextran matrix of a surface plasmon resonance sensor chip," Analytical Biochemistry, vol. 280, no. 1, pp. 29-35, 2000.

[18] D. Beccati, K. M. Halkes, G. D. Batema et al., "SPR studies of carbohydrate-protein interactions: signal enhancement of low-molecular-mass analytes by organoplatinum(II)-labeling," ChemBioChem, vol. 6, no. 7, pp. 1196-1203, 2005.

[19] H. Zhang, L. Yang, B. Zhou et al., "Investigation of biological cell-protein interactions using SPR sensor through laser scanning confocal imaging-surface plasmon resonance system," Spectrochimica Acta-Part A: Molecular and Biomolecular Spectroscopy, vol. 121, pp. 381-386, 2014.

[20] M. Beseničar, P. Maček, J. H. Lakey, and G. Anderluh, "Surface plasmon resonance in protein-membrane interactions," Chemistry and Physics of Lipids, vol. 141, no. 1-2, pp. 169-178, 2006.

[21] H. Miyoshi, N. Suehiro, K. Tomoo et al., "Binding analyses for the interaction between plant virus genome-linked protein (VPg) and plant translational initiation factors," Biochimie, vol. 88, no. 3-4, pp. 329-340, 2006.

[22] B. Song, D. Li, W. Qi, M. Elstner, C. Fan, and H. Fang, "Graphene on $\mathrm{Au}(111)$ : a highly conductive material with excellent adsorption properties for high-resolution bio/nanodetection and identification," ChemPhysChem, vol. 11, no. 3, pp. 585-589, 2010.

[23] M. B. Hossain and M. M. Rana, "An effective compact-FDTD wideband modeling of graphene conductivity," in Proceedings of 
the IEEE International Conference on Electrical Engineering and Information Communication Technology (ICEEICT '15), pp. 1-3, IEEE, Dhaka, Bangladesh, May 2015.

[24] S. H. Choi, Y. L. Kim, and K. M. Byun, "Graphene-on-silver substrates for sensitive surface plasmon resonance imaging biosensors," Optics Express, vol. 19, no. 2, pp. 458-466, 2011.

[25] R. L. Earp Jr., Multiwavelength surface plasmon resonance sensor designs for chemical and biochemical detection [Ph.D. thesis], 1998.

[26] L. Diéguez, N. Darwish, M. Mir, E. Martínez, M. Moreno, and J. Samitier, "Effect of the refractive index of buffer solutions in evanescent optical biosensors," Sensor Letters, vol. 7, no. 5, pp. 851-855, 2009.

[27] L. Diéguez, D. Caballero, J. Calderer, M. Moreno, E. Martínez, and J. Samitier, "Optical gratings coated with thin $\mathrm{Si}_{3} \mathrm{~N}_{4}$ layer for efficient immunosensing by optical waveguide lightmode spectroscopy," Biosensors, vol. 2, no. 2, pp. 114-126, 2012.

[28] R. Boruah, D. Mohanta, A. Choudhury, P. Nath, and G. A. Ahmed, "Surface plasmon resonance based protein biosensing using a Kretschmann configured double prism arrangement," IEEE Sensors Journal, vol. 15, no. 12, pp. 6791-6796, 2015.

[29] R. Otupiri, E. K. Akowuah, S. Haxha, H. Ademgil, F. AbdelMalek, and A. Aggoun, "A novel birefrigent photonic crystal fiber surface plasmon resonance biosensor," IEEE Photonics Journal, vol. 6, no. 4, 2014.

[30] A. Ishimaru, Electromagnetic Wave Propagation, Radiation, and Scattering, Prentice Hall, Upper Saddle River, NJ, USA, 1991.

[31] L.-X. Dong and Q. Chen, "Properties, synthesis, and characterization of graphene," Frontiers of Materials Science in China, vol. 4, no. 1, pp. 45-51, 2010.

[32] L.-J. Wang, G. Cao, T. Tu et al., "A graphene quantum dot with a single electron transistor as an integrated charge sensor," Applied Physics Letters, vol. 97, no. 26, Article ID 262113, 2010. 


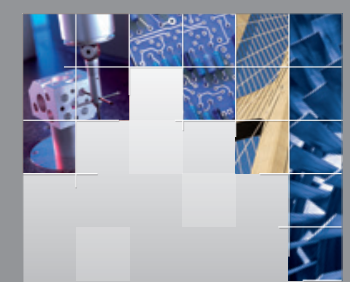

\section{Enfincering}
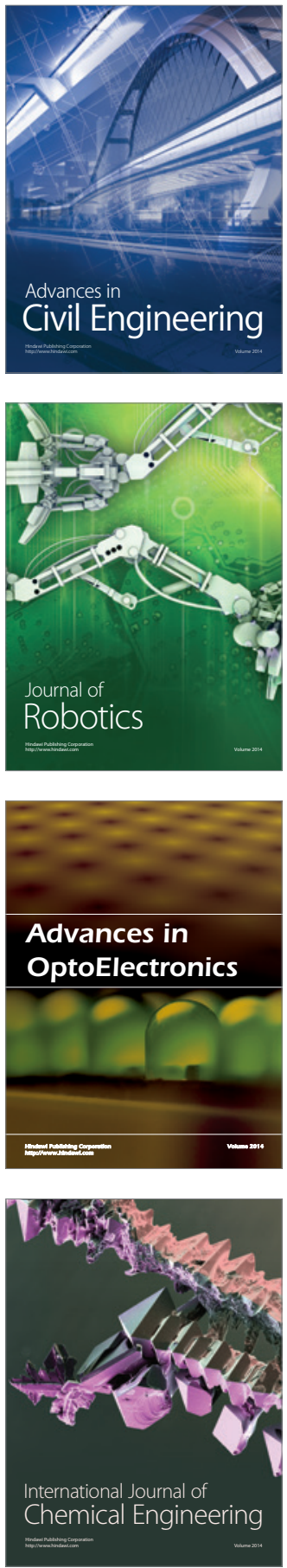

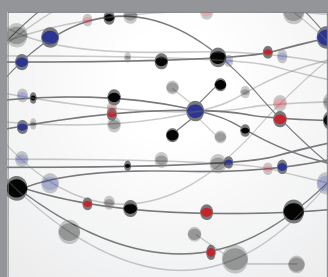

The Scientific World Journal

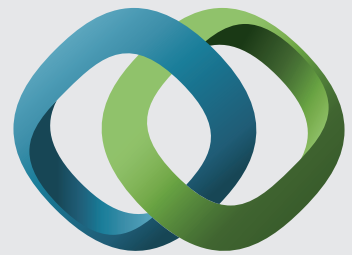

\section{Hindawi}

Submit your manuscripts at

http://www.hindawi.com
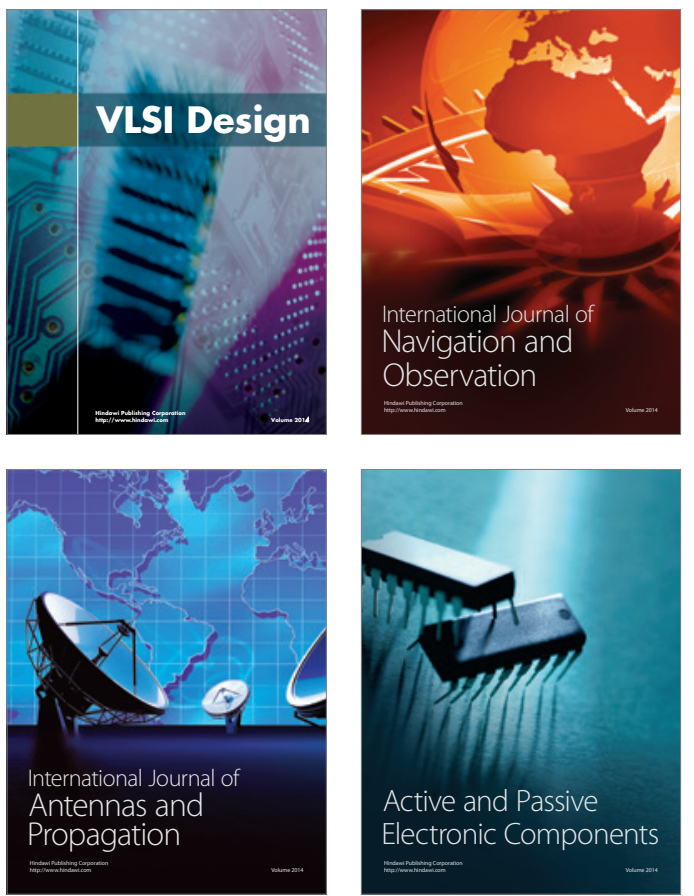
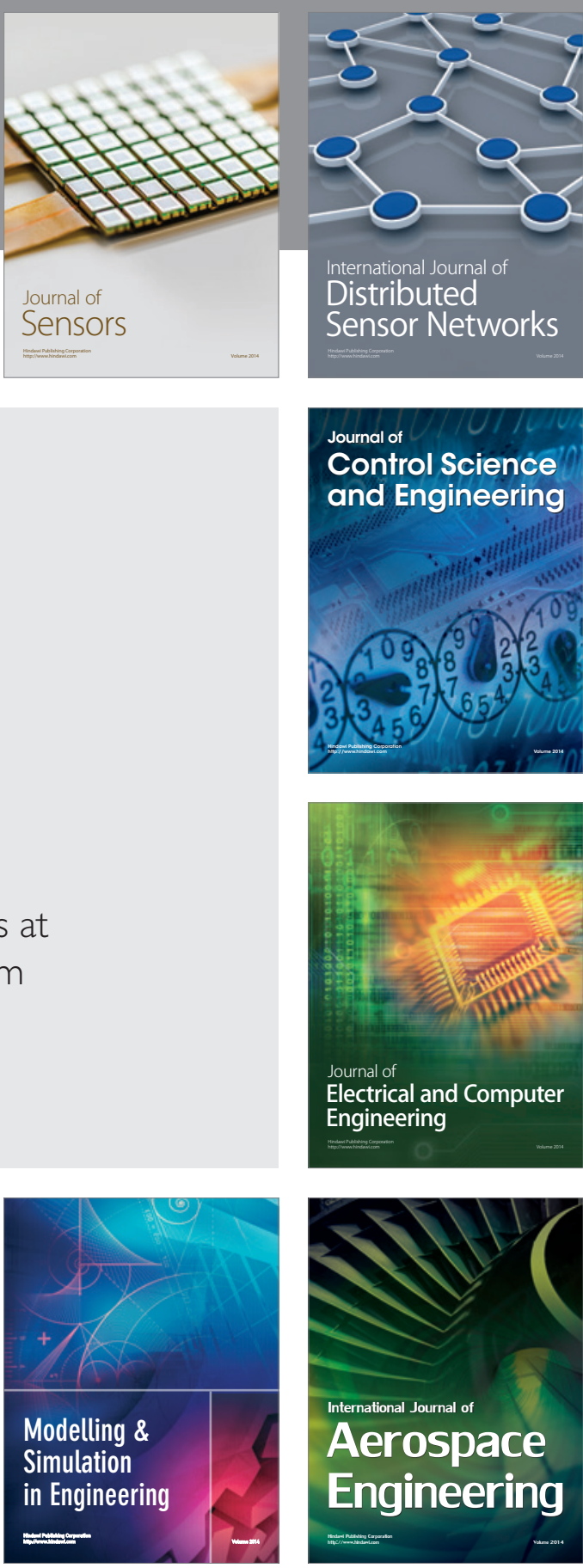

International Journal of

Distributed

Sensor Networks

Journal of

Control Science

and Engineering
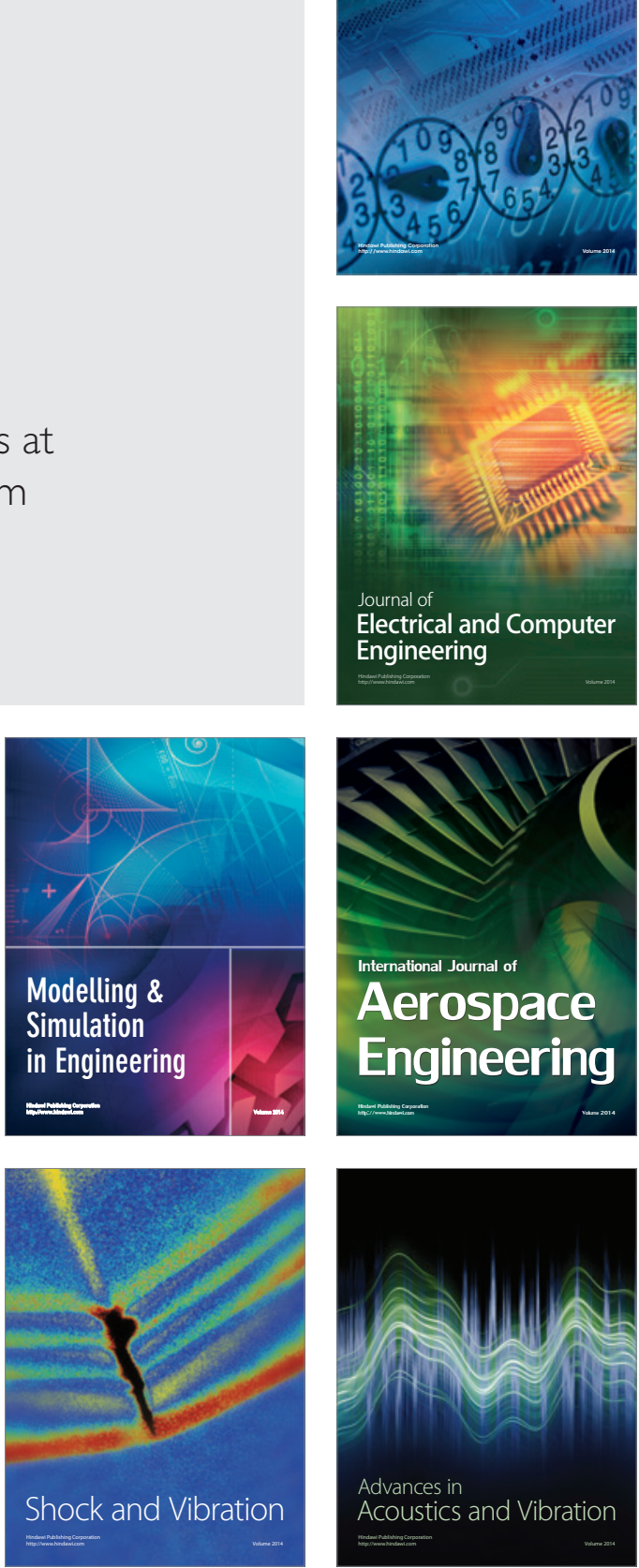